\title{
QUANTITATIVE RELAXATION TEMPLATES FOR THE HUMAN BRAIN AT 3T
}

\author{
Fang Cao, Olivier Commowick, Camille Maumet, Christian Barillot \\ VisAGeS U746 INSERM/INRIA, IRISA UMR CNRS 6074, Rennes, France
}

\begin{abstract}
The development of brain Magentic Resonance Imaging (MRI) is driving increasing demand for quantitative measurements. Quantitative MRI (qMRI) templates of relaxation times and proton density can be of particular interest for dedicated clinical applications such as characterizing brain tissue abnormalities, as well as general research purposes. In this paper, we have developed 3D qMRI statistical templates consisting of $T_{1}, T_{2}, T_{2}^{*}$ and $\rho^{*}$ maps from the human brain at 3T. The qMRI templates were built from a population of 20 normal controls, for which individual maps were estimated in a robust manner, accounting for acquisition artifacts and expected relationships between the relaxometry parameters. For validation, we fed the qMRI templates into a realistic MRI simulator to synthesize MR-weighted images, and compared these images with the real MR acquisitions. High correlation coefficients $(>0.80)$ show that the developed qMRI templates can be used as input dataset for MRI simulation community, which may be of great interest to clinical neuroscience field.
\end{abstract}

Index Terms $-T_{1} ; T_{2} ; T_{2}^{*}$; relaxation time; effective proton density $\rho^{*}$; brain template; MRI simulation

\section{INTRODUCTION}

Objective measurements from quantitative MRI have a great importance in characterizing diseased brain tissues and have attracted wide interest [1]. They can be used as brain structures fingerprints for characterization of pathologies as suggested recently in [2]. The availability of relaxometry templates (relaxation times and proton density) could be of great interest for MRI simulations. It would allow to generate populations of images with realistic variations of contrast and intensities, possibly with the addition of brain pathologies (i.e. tumor and lesions), to study brain disease evolutions. In addition, having a relaxometry template would be of great use to quantitatively study the influence on brain tissues of a patient suffering from neurodegenerative diseases.

Recent works have studied the construction of anatomical atlases from different points of view. First, we may use one typical brain image or model, either to generate typical brain acquisitions ${ }^{1}$ [3] or as a reference for studies such

\footnotetext{
Thanks to the ANR-VIP project and ARSEP France for funding.

${ }^{1}$ http: / / wWw.bic.mni.mcgill.ca/brainweb
}

as the Talairach atlas [4]. However, the large variability in brain anatomy makes it difficult to construct a representative atlas from a single brain image and therefore several groups studied the construction of average atlases [5,6]. All these approaches, although relying on different registration algorithms (pairwise or groupwise registration), have the objective of creating an unbiased average anatomy of the brain from a database of subjects.

Based on such template creation methods, several atlases of the brain have been proposed recently. Mazziotta et al. [7] proposed the MNI152 atlas built from the ICBM consortium database, which consists of an average T1-w image and associated segmentations. From this same database, a larger atlas ICBM $452^{2}$ was built to serve as an anatomical standard. The LPBA40 atlas [8] consists of an average anatomical T1w image and gray matter parcellation and is often used for automatic brain segmentation. Finally, the SRI24 atlas was recently developed [9], grouping T1-w, segmentation and diffusion tensor information into one single multi-channel atlas. However, all recently proposed atlases of the human brain do not include quantitative features of the human brain such as those extracted from relaxometry.

We propose a novel method to create qMRI templates of $T_{1}, T_{2}, T_{2}^{*}$ and $\rho^{*}$. It integrates a robust estimation of individual relaxometry maps, accounting for acquisition artifacts and expected relationships between different relaxometry parameters. At the same time, a high-resolution T1-w template was created from the individual MPRAGE images. The relaxation maps were then registered on the T1-w template space to compute the qMRI templates. We validate the constructed atlas using simulation tools, and finally present the application of our atlas to generate a population of subjects with different modalities.

\section{MATERIAL AND METHODS}

\subsection{Data Acquisition}

Whole-brain MR images were acquired on 20 healthy subjects ( 7 male, 13 female, mean age $=32.0 \pm 15.1$ y.o.). All imaging experiments for this study were performed on a $3 \mathrm{~T}$ Siemens Verio (VB17) scanner with a 32-channel head coil.

\footnotetext{
${ }^{2}$ http://www.Ioni.ucla.edu/Atlases
} 
The acquisition protocol included $T_{1}, T_{2}, T_{2}^{*}$ relaxometry sequences and a 3D MPRAGE T1-w sequence. For the $T_{1}$ relaxometry measurement, we used two Spoiled GRE sequences with fixed repetition time TR $=15 \mathrm{~ms}$ and flip angles $\theta=\left[5.0^{\circ}, 30^{\circ}\right]$. For the $T_{2}$ relaxometry sequence, seven echoes were acquired using SE sequence with $\mathrm{TR}=4530$ $\mathrm{ms}$ and $\mathrm{TE}=[13.8,27.6,41.4,55.2,69.0,82.8,96.6] \mathrm{ms}$. For the $T_{2}^{*}$ relaxometry sequence, five echoes were acquired using GRE sequence with $\mathrm{TE}=[4.36,11.9,19.44,26.98$, $34.52] \mathrm{ms}$. All relaxometry sequences have an image size of $192 \times 192 \times 44$ and a voxel size of $1.3 \times 1.3 \times 3 \mathrm{~mm}^{3}$. The MPRAGE sequence is a $3 \mathrm{D}$ high resolution T1-w sequence. The acquisition parameters were TR/TE/TI $=1900 / 2.98 / 900$ $\mathrm{ms}$, image size $=256 \times 256 \times 160$, voxel size $=1 \times 1 \times 1 \mathrm{~mm}^{3}$.

For validation purposes, T1-w and T2-w/PD-w sequences were acquired. The T1-w image was acquired with an SE sequence. The acquisition parameters were TR/TE $=500 / 8.4$ $\mathrm{ms}$, image size $=256 \times 256 \times 44$, voxel size $=1 \times 1 \times 3 \mathrm{~mm}^{3}$. The PD-w and T2-w images were acquired with a Turbo Spin Echo sequence. Its parameters were TR/TE $=6530 \mathrm{~ms}, \mathrm{TE}=$ $[9.4,84] \mathrm{ms}$, image size $=192 \times 256 \times 44$, voxel size $=1 \times 1 \times 3$ $\mathrm{mm}^{3}$.

\subsection{QMRI Statistic Template Construction}

Fig. 1 illustrates the overall workflow for construction of the 3D qMRI template from 20 subjects. To compensate for between-scans subject motion, a six-parameter rigid-body registration of each relaxometry map $\left(T_{1}, T_{2}, T_{2}^{*}\right.$ and $\left.\rho^{*}\right)$ on the MPRAGE image was carried out for each subject based on normalised mutual information.

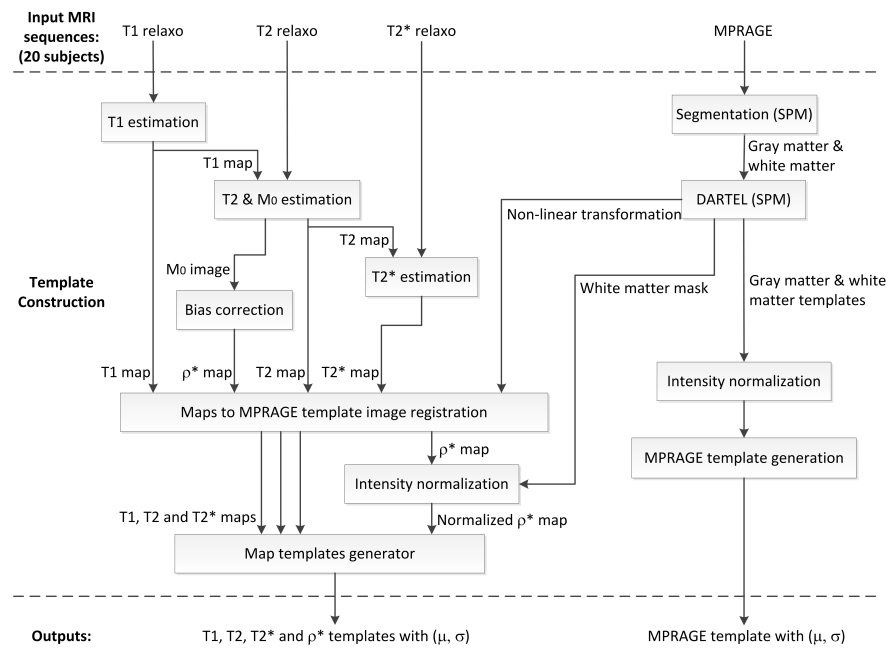

Fig. 1. Brain qMRI template generator.

Our construction method is composed of two parts. (1) From the $T_{1}$-w images (right part in Fig. 1), we used the Matlab toolbox SPM8 ${ }^{3}$ to generate an MPRAGE average tem-

\footnotetext{
${ }^{3}$ http: //www.fil.ion.ucl.ac.uk
}

plate. The anatomical image of each subject was segmented using unified segmentation [10]. Spatial normalisation across subjects was then carried out with DARTEL [11]. It should be noted that we only applied DARTEL registration to white matter and gray matter as CSF segmentation is not always accurate. Then, we built the MPRAGE template by calculating the mean and variance on the group of subjects. This template was used to create the anatomical model in order to serve as a common space for all subjects. (2) Starting from the $T_{1}, T_{2}$, $T_{2}^{*}$ relaxometry sequences (left part in Fig. 1), we performed map estimations for each subject and transformed them to the MPRAGE template. The estimations of maps were performed successively, and all estimations were modeled as non-linear regression problems with constraints.

The estimation of $T_{1}$ considered potential small uncertainties or variations of the flip angles when the sequences were acquired. To ensure a smooth variation of the flip angles, we added an L-2 regularisation term in the energy function. The $T_{1}$ value was restricted in the range of $[1,5000] \mathrm{ms}$ based on prior knowledge [12]. The problem amounted to minimize the energy function for all voxels $v$ in the whole volume $V$ :

$$
E_{T_{1}}=\int_{V}\left\|F_{1}\left(T_{1}, M_{0}, \theta\right)-S_{T_{1}}\right\|^{2} \mathrm{~d} v+\lambda \int_{V}\|\nabla \theta\|^{2} \mathrm{~d} v
$$

where $M_{0}$ is proportional to the equilibrium value of the magnetization, $\theta$ is the vector of the two flip angles, $S_{T_{1}}$ denotes the vector with the acquired two $T_{1}$ relaxometry signals, and $F_{1}\left(T_{1}, \theta\right)$ is the vector with the two simulated signals obtained from the equation [13]

$$
F_{1}\left(T_{1}, M_{0}, \theta\right)=\frac{M_{0}\left(1-\mathrm{e}^{\frac{-T R}{T_{1}}}\right) \sin \theta}{1-\mathrm{e}^{\frac{-T R}{T_{1}}} \cos \theta}
$$

The optimization process was performed iteratively on the two terms in equation (1) until convergence. We performed an iterative minimization scheme, starting from minimizing the first term using fixed initial values $T_{1}=1, M_{0}=0$ and $\theta=\left[\begin{array}{ll}\theta_{1} & \theta_{2}\end{array}\right]$. Then, the second term was optimized via Gaussian smoothing.

The estimations of $T_{2}$ and $T_{2}^{*}$ are similar optimization problems (shown here for the $T_{2}$ maps):

$$
E_{T_{2}}=\int_{V}\left\|F_{2}\left(T_{2}, M_{0}\right)-S_{T_{2}}\right\|^{2} \mathrm{~d} v
$$

where $S_{T_{2}}$ denotes the vector with the acquired multiple $T_{2}$ relaxometry signals and $F_{2}\left(T_{2}, M_{0}\right)$ is the vector with the simulated signals following the exponential equation [13]

$$
F_{2}\left(T_{2}, M_{0}\right)=M_{0} \mathrm{e}^{\frac{-T E}{T_{2}}}
$$

Besides the constraints $[0,1000] \mathrm{ms}$ for both $T_{2}$ and $T_{2}^{*}$, we use a sequential design to introduce extra constraints $T_{2} \leq T_{1}$ and $T_{2}^{*} \leq T_{2}$ [14] into the $T_{2}$ and $T_{2}^{*}$ estimation respectively. 
Effective proton density $\rho^{*}$ [15] was calculated from the $M_{0}$ in the $T_{2}$ estimation, as the $T_{2}$ relaxometry sequence has the largest number of echoes among all relaxometry sequences and thus potentially the highest accuracy. The $\rho^{*}$ map was drawn from $M_{0}$ by applying the formula $M_{0} /\left(1-\mathrm{e}^{\frac{-T R}{T_{1}}}\right)$ and by adjusting receive sensitivity using the bias correction in SPM [10]. Moreover, the values of $\rho^{*}$ vary from subject to subject, which is not the case for $T_{1}, T_{2}$ and $T_{2}^{*}$ maps. Therefore, we integrated the intensity normalization for $\rho^{*}$ map into the workflow. An iterative linear least square method was used to center the $\rho^{*}$ values in the areas of gray matter and white matter across all subjects.

Finally, we modeled the voxel values using a univariate Gaussian distribution that fits well the real measures, and calculated the means and standard deviations on the group of subjects to generate the relaxometry templates.

\subsection{Dataset Description}

All data were saved as Nifti files. The $3 \mathrm{D} T_{1}, T_{2}$ and $T_{2}^{*}$ map templates, the 3D T1-W MPRAGE template together with the DARTEL template were included in the package. All images have a dimension of $181 \times 217 \times 181$ with the voxel size of $1 \times 1 \times 1 \mathrm{~mm}^{3}$. The data can be loaded using SPM, Mricron or MedInria. A readme file is also given in the package.

\subsection{QMRI Statistic Template Validation}

We used a MRI simulator ${ }^{4}$ based on Bloch equations [16] to validate the estimated qMRI templates. The validation process included two parts. (1) The first part was to acquire different MR sequences on the same population. We used T1-w SE and T2-w/PD-w sequences and applied spatial normalization to the sequences in order to match the existing MPRAGE T1-w template. The template for each weighted image was generated after applying intensity normalisation across all subjects. These T1-w, T2-w and PD-w image templates were used as reference datasets (the real acquisitions) for the template validation. (2) The second part for the validation was to use the qMRI mean templates as inputs of the MRI simulator to generate the simulated T1-w, T2-w and PD-w images. The simulations were done with the same sequences and parameters as the real acquisitions. Assuming that the templates are ideally constructed and the simulations are perfect, a linear relationship should be obtained between the simulated and the real image. As a validation of the template coherence, we calculated the correlation coefficient $R$ in the brain to measure the strength of linear dependence between the simulated images and the corresponding real acquisitions. A value of $R=1$ implies a perfect simulation.

\footnotetext{
${ }^{4}$ SimuBloch v0.3 http://vip.creatis. insa-lyon.fr
}

\section{RESULTS AND DISCUSSION}

QMRI Template Visualization: Fig. 2 presents the mean and standard deviation templates of $T_{1}, T_{2}, T_{2}^{*}$ and $\rho^{*}$ maps. It shows the homogenization in gray matter and white matter regions on the mean templates. Averaging over multiple subjects enhances the visibility of fine structures in deep brain.

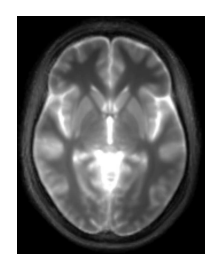

(a) $T_{1}$ map, $\mu$ [134, 3612] $\mathrm{ms}$

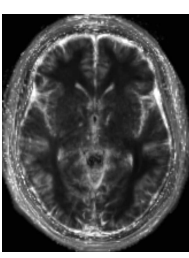

(b) $T_{1}$ map, $\sigma$ $[0,1159] \mathrm{ms}$

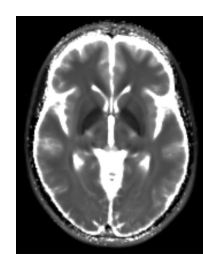

(c) $T_{2}$ map, $\mu$ $[42,163] \mathrm{ms}$

(d) $T_{2}$ map, $\sigma$ $[0,149] \mathrm{ms}$ $\min$

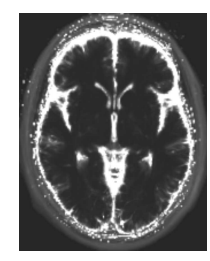

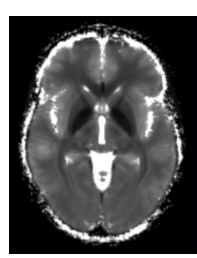

(e) $T_{2}^{*}$ map, $\mu$ $[20,116] \mathrm{ms}$

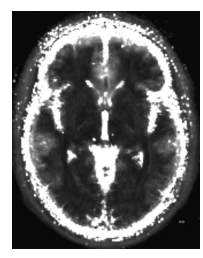

(f) $T_{2}^{*}$ map, $\sigma$ $[0,31] \mathrm{ms}$

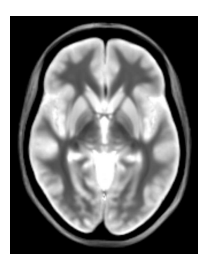

(g) $\rho^{*}$ map, $\mu$ $[48,106]$

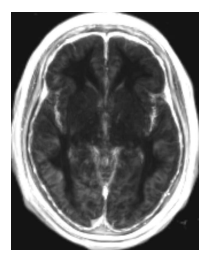

(h) $\rho^{*}$ map, $\sigma$ $[0,31]$
Fig. 2. QMRI mean and standard deviation templates. [min, $\max$ ] give the range of the colorbar for each template.

Validation: In Fig. 3, the first column gives the weighted mean templates (real acquisitions) and the second column gives the simulated weighted images. In the brain, the correlation coefficients $R$ between the real acquisitions and the simulated images are 0.96 for the T2-w sequence and 0.94 for the PD-w sequence. Both T2-w and PD-w simulations show a strong positive correlation (the $R$ values are close to 1 ) between the simulated sequence and the real acquisition. The value of $R$ for the T1-w sequence is equal to 0.82 , which is relatively lower than these for the T2-w and PD-w sequences. This may be due to the $B_{1}$ inhomogeneities in the $T_{1}$ map that needs further investigation.

Diverse MR Sequence Generation: A direct application of the qMRI templates is to simulate realistic MR weighted sequences over a population. We took the mean and standard deviation templates (Fig. 2) and used a random value $x$ to generate a sample from the Normal distribution $(\mu+x \sigma)$ for each of the relaxometry maps. The generation process was repeated twice to provide various contrasts and intensities in the sequence images. In the third and fourth columns of Fig. 3, the values of $x$ for the sample maps $\left(\mathrm{T} 1, \mathrm{~T} 2, \mathrm{~T} 2 *, \rho^{*}\right)$ are $(0.22,1.38,0.90,-0.98)$ for \#1 and $(-0.75,-0.36,3.58,0.07)$ for \#2. These samples of maps were used as input modalities to feed the MRI simulator in order to synthesize different MR sequences, such as T1-w, T2-w and PD-w images (Fig. 3(g- 


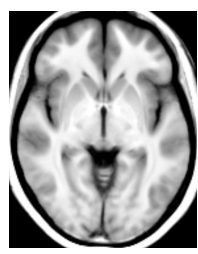

(a) T1-w, real

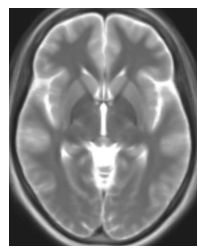

(b) T2-w, real

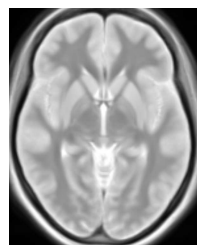

(c) PD-w, real

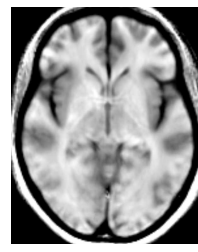

(d) T1-w, simu

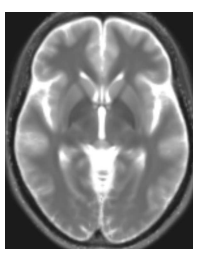

(e) T2-w, simu

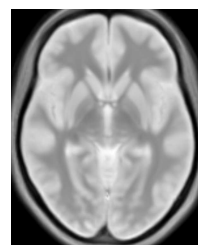

(f) PD-w, simu

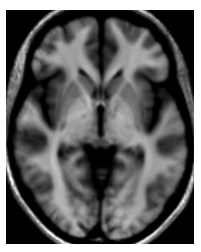

(g) T1-w, \#1

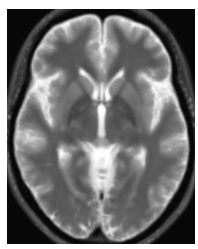

(h) T2-w, \#1

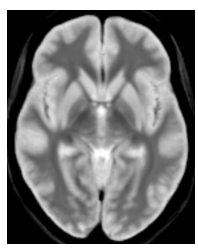

(i) PD-w, \#1

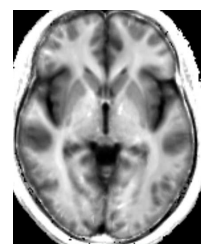

(j) T1-w, \#2

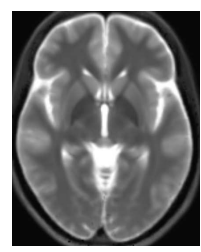

(k) T2-w, \#2

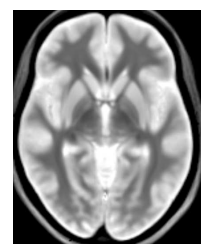

(1) PD-w, \#2
Fig. 3. Validation and diverse MR sequences simulation.

1)). The results show that using quantitative atlas allows us to generate images with realistic variations of contrast and intensity, thereby enabling to quantify the sensitivity of an algorithm to these variations.

\section{CONCLUSIONS}

We have proposed a 3D qMRI template generator from healthy human brain at 3T. The templates were constructed using the relaxometry and MPRAGE sequences on 20 normal subjects. The mean templates showed a clear enhancement of the visibility of fine structures in deep brain. We have validated the templates by showing high correlation coefficients $(>0.80)$ between the simulated weighted images and the real acquisitions. We have also demonstrated the effectiveness of using these templates to simulate MR scans. Our qMRI templates can complement the lack of quantitative data in MRI simulation field. A future work may be to create disease-specific brain templates, in conditions like Multiple Sclerosis, which may aid in crucial neuroimage analysis. The qMRI templates are available at https://www.irisa.fr/visages/download to serve as a quantitative dataset for neuroimaging studies.

\section{REFERENCES}

[1] J.B.M. Warntjes, O.D. Leinhard, et al., "Rapid magnetic resonance quantification on the brain: Optimization for clinical usage," MRM, vol. 60, no. 2, pp. 320-329, 2008.
[2] D. Ma, V. Gulani, et al., "Magnetic resonance fingerprinting," Nature, vol. 495, no. 7440, pp. 187-192, Mar. 2013.

[3] B. Aubert-Broche, M. Griffin, et al., "Twenty new digital brain phantoms for creation of validation image data bases," IEEE Trans. Med. Imaging, vol. 25, no. 11, pp. 1410-1416, 2006.

[4] J. Talairach and T. Tournoux, Co-planar Stereotaxic Atlas of the Human Brain: 3-Dimensional Proportional System - An Approach to Cerebral Imaging, New York: Thieme Medical Publishers, 1988.

[5] S. Joshi, B. Davis, et al., "Unbiased diffeomorphic atlas construction for computational anatomy," Neuroimage, vol. 23 Suppl 1, pp. S151-60, 2004.

[6] K.K. Bhatia, P. Aljabar, et al., "Groupwise combined segmentation and registration for atlas construction," in MICCAI, 2007.

[7] J. Mazziotta, A. Toga, et al., "A probabilistic atlas and reference system for the human brain: International Consortium for Brain Mapping (ICBM),' Philos Trans $R$ Soc Lond B Biol Sci, vol. 356, pp. 1293-1322, 2001.

[8] D.W. Shattuck, M. Mirza, et al., "Construction of a 3D probabilistic atlas of human cortical structures," Neuroimage, vol. 39, pp. 1064-1080, 2008.

[9] T. Rohlfing, N.M. Zahr, et al., "The SRI24 multichannel atlas of normal adult human brain structure," $H B M$, vol. 31, pp. 798-819, 2010.

[10] J. Ashburner and K.J. Friston, "Unified segmentation," NeuroImage, vol. 26, pp. 839-851, 2005.

[11] J. Ashburner, "A fast diffeomorphic image registration algorithm.," Neuroimage, vol. 38, no. 1, pp. 95-113, 2007.

[12] C. Dagia and M. Ditchfield, "3T MRI in paediatrics: challenges and clinical applications," Eur J Radiol, vol. 68, no. 2, pp. 309-319, 2008.

[13] P. Tofts, Quantitative MRI of the Brain: Measuring Changes Caused by Disease, J. Wiley \& Sons, 2003.

[14] M.A. Heinrichs and A. Siemens, Magnets, Spins, and Resonances: An Introduction Into the Basics of Magnetic Resonance Imaging, Siemens, 1992.

[15] N. Weiskopf, J. Suckling, et al., "Quantitative multiparameter mapping of R1, PD*, MT, and R2* at 3T: a multi-center validation," Front. Neurosci., vol. 7, no. 95, 2013.

[16] F. Bloch, "Nuclear induction," Physical Review, vol. 70, pp. 460-474, 1946. 